\title{
A New Surgical Approach for Direct Acoustic Cochlear Implant: A Temporal Bone Study
}

\author{
Luca Bruschini · Francesca Forli · Andrea De Vito · Stefano Berrettini \\ ENT Audiology and Phoniatry Unit, University Hospital of Pisa, Pisa, Italy
}

Objectives. The direct acoustic cochlear implant (DACI) is among the latest developments in the field of implantable acoustic prostheses. The surgical procedure requires a mastoidectomy and a posterior-inferior tympanotomy, with access to the facial recess at the level of the oval window, in a complex and lengthy surgical approach. Here, we report a new and considerably shorter surgical approach.

Methods. The new approach involves positioning of artificial incus above the oval window through the superior-anterior tympanotomy. We performed DACI placement in temporal bone specimens $(n=5)$ to assess the feasibility of the new approach.

Results. The average time for the DACI implant in the temporal bones was only 112 minutes (range, 94 to 142 minutes) and there was little clinical risk associated with the procedure. Access was easy and drilling was minimal.

Conclusion. Our approach simplified the surgical procedure and consequently reduced the time required for DACI placement.

Keywords. Acoustics; CODACS; Tympanotomy; Prostheses and Implants

\section{INTRODUCTION}

Implantable acoustic prostheses can be classified into three categories according to the mechanism of inner ear stimulation. The first category comprises middle ear implants (MEI), which transmit an amplified vibration to the cochlea via the ossicular chain [1-7]. A second category comprises bone-conduction implants that transmit vibration via the bone, excluding the middle and outer ear. The third category comprises prostheses that stimulate the cochlea by vibrating the perilymph through the oval window (OW) for severe-to-profound mixed hearing loss, and are designated as direct acoustic cochlear implants (DACI). The concept was presented initially by Hausler et al. [8] in 2008 as a

\footnotetext{
- Received November 8, 2015

Revised December 12, 2015

Accepted December 15, 2015

- Corresponding author: Luca Bruschini

UO Otorinolaringoiatria, Azienda Ospedaliera Universitaria Pisana, Via

Paradisa 2, 56127 Pisa, Italy

Tel: +39-347-3558587, Fax: +39-050-997495

E-mail: 1.bruschini@gmail.com
}

percutaneous system, called DACS, and was further developed into the transcutaneous Codacs system (Cochlear Ltd., Sydney, Australia), which was investigated in two clinical trials $[9,10]$ and is currently commercially available.

The Codacs system comprises an implantable part, consisting of a receiver coil, the implant electronics, and the electromagnetic actuator, which is held in place by a fixation system, and an externally worn part that is a behind-the-ear sound processor with a radio-frequency coil, i.e., the Cochlear Nucleus CP810 sound processor with modified firmware that facilitates acoustic signal processing. The actuator has a terminal-referred to as the 'artificial incus'-which is similar to the size of the long process of the incus. The fixation system is fastened to the mastoid cortex with screws, with the ball joint allowing movement and locking of the actuator into the correct position [11]. The artificial incus should be positioned in such a way that it is aligned to the level of the natural incus, above the OW, while avoiding contact with the surrounding tissues and bony structures.

The surgical technique used to insert this system when it was first developed was similar to that used for the minimally inva-

Copyright @ 2016 by Korean Society of Otorhinolaryngology-Head and Neck Surgery.

This is an open-access article distributed under the terms of the Creative Commons Attribution Non-Commercial License (http://creativecommons.org/licenses/by-nc/4.0)

which permits unrestricted non-commercial use, distribution, and reproduction in any medium, provided the original work is properly cited. 

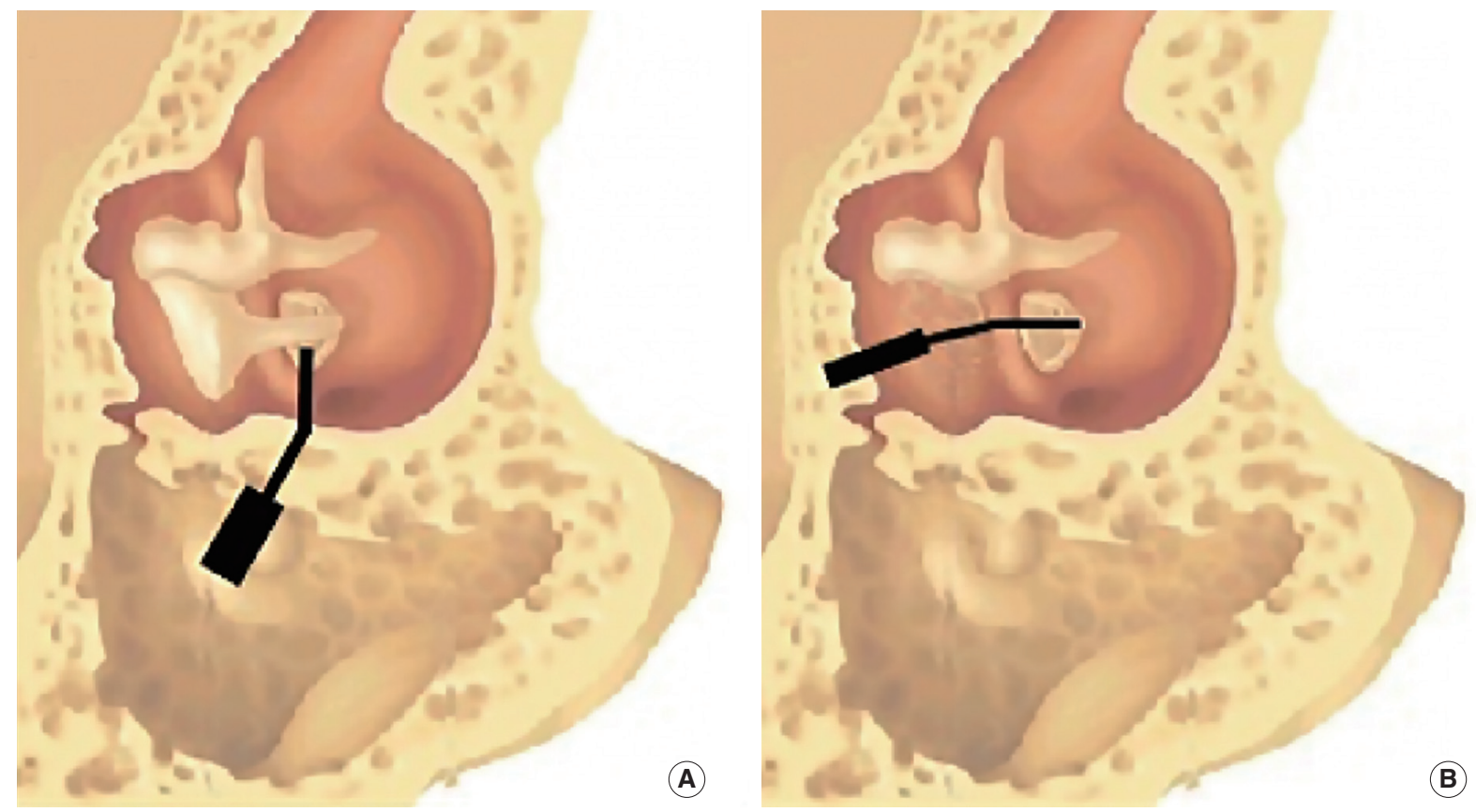

Fig. 1. The two surgical procedures. (A) Haeusler and Lenarz procedure: device in the mastoid with the artificial incus, through the posterior tympanotomy, orthogonal at the incus. (B) Our procedure: device in the mastoid with the artificial incus, through the anterior-superior tympanotomy, in place of the incus.

sive cochlear implant technique described by Hausler [12]. The surgical procedure included a small mastoidectomy, limited to a retro-canal tunnel and a posterior-inferior tympanotomy, with access to the facial recess at the level of the OW. This access allowed introduction of the fixation system and the actuator, positioning of the artificial incus, and execution of a stapedotomy. In the operations performed by Hausler et al. [8] in four patients with severe-to-profound hearing loss due to otosclerosis, two stapes prosthesis were used: one anchored to the artificial incus and the other to the natural incus. Hausler et al. [8] reported good functional results; in particular, a gain in the postoperative air conduction threshold with the implant inactive and the piston anchored to the natural incus (range, 14 to $28 \mathrm{~dB} ; \mathrm{n}=4$ ) and a markedly higher gain with the implant active (range, 41 to 62 $\mathrm{dB} ; \mathrm{n}=4$ ). The intervention took a relatively long time (range, 2.5 to 5 hours; $n=4$ ). It was technically complex, but without any noticeable intra- or postoperative complications. The risk of cochlear damage was comparable to that of a classical stapedotomy [8]. In 2012, Zwartenkot et al. [13] compared the results of

\section{H I G H L I G H T T S}

- The direct acoustic cochlear implant (DACI) is a innovative and useful implantable prosthesis.

- We evaluated a new surgical procedure to place the DACI.

- We proposed to implant the DACI through the superior-anterior tympanotomy, in place of the incus. these four patients to those obtained with nine Vibrant Soundbridge (VSB) implants and nine middle ear transducer (MET) implants. The DACS system demonstrated a better amplification capacity than the VSB and was similar or superior to the MET, but the surgery required was considered more invasive, as it included opening of the vestibule.

More recently, Lenarz et al. $[9,10]$ published the results of two multicenter studies with the DACI system. The surgical procedure was based on the approach used by Hausler et al. [8], but none of the ossicular chains was reconstructed with the second stapes prosthesis. Similar to Hausler et al. [8], Lenarz et al. $[9,10]$ placed the device in the mastoid, with the tip on the footplate of the stapes, through the posterior tympanotomy (Fig. 1). This posterior approach to the middle ear leads to a reduced view of the stapes footplate, the need to expose the facial nerve, and sometimes the need to drill into a part of the posterior canal wall. Moreover, the reduced view of the stapes footplate obligates the surgeon to make a hole in the stapes footplate before placing the device. Therefore, the vestibule stays open for about 30 minutes to 1 hour. The relative complexity of the surgery was highlighted and, in the first trial, the average surgical time was 4 hours and 52 minutes ( $\mathrm{SD}=1$ hour and 26 minutes), including intraoperative testing with a laser Doppler vibrometer.

In consideration of the technical difficulties encountered with the placement of this system, which are reported by all surgeons, we sought to find a simpler implantation procedure. Here, we report the experimentally validated feasibility of a new, simpler, and faster approach for DACI placement. 

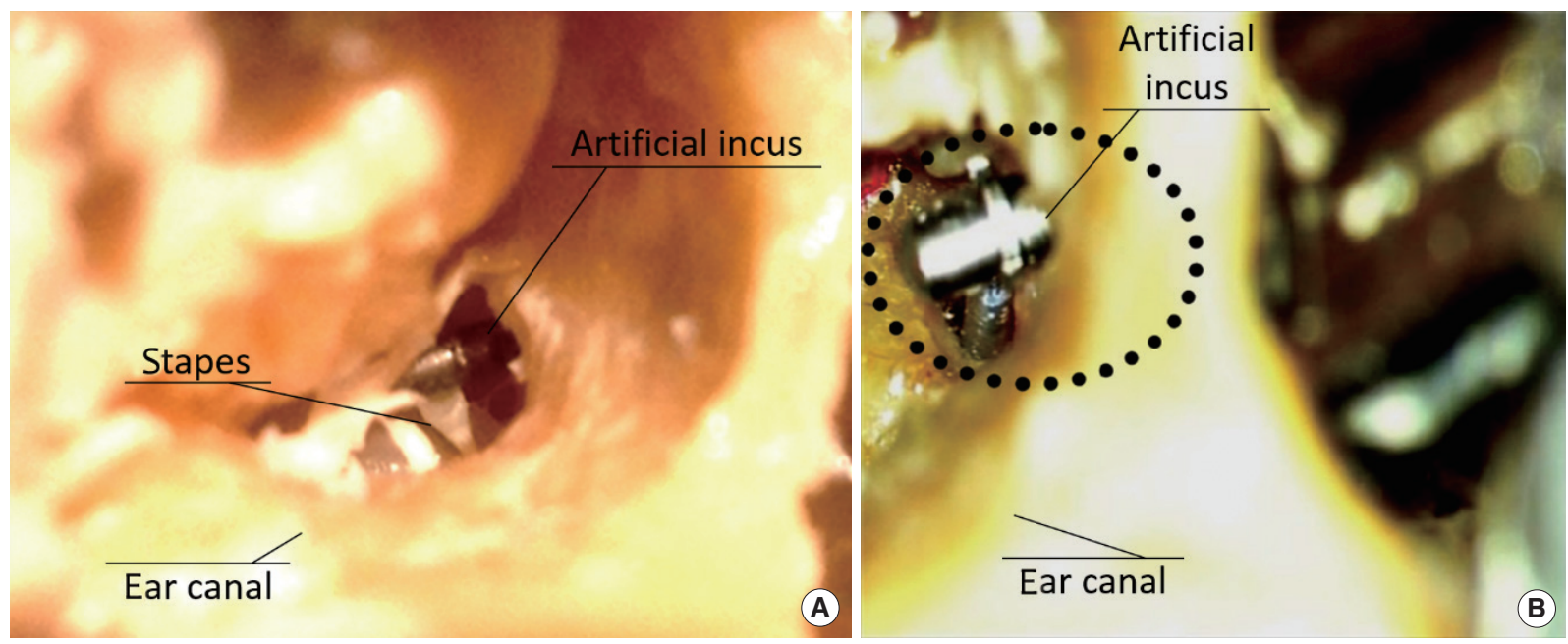

Fig. 2. The stapes and artificial incus seen through the external auditory canal. (A) The artificial incus is in the same position as the natural incus, over the stapes. (B) The artificial incus is in the same position as the natural incus, with the piston positioned (dotted circle).

\section{MATERIALS AND METHODS}

We developed a new surgical procedure for implanting the DACI on five frozen temporal bone specimens in the laboratory of the ENT unit of the University Hospital of Pisa, which was equipped with a surgical microscope (Leica M400; Leica, Wetzlar, Germany), as well as surgical drill and instruments. The human anatomical samples consisted of the bone and the soft tissue of the temporal and mastoid region. Ethical approval was waived by the University of Pisa.

Before the intervention, a high-resolution computed tomography (CT) scan of the bones was performed to establish the dimensions of the mastoid and the epitympanic region. The CT scan, with a $0.6-\mathrm{mm}$ section thickness, allowed axial, coronal, and sagittal reconstruction of temporal bone images. All the surgical procedures were performed by a senior surgeon.

\section{Surgical procedure}

The dissection begins with the canal incision of a tympanomeatal flap, its detachment, and drilling into a part of the epitympanic frame sufficiently large to allow access to the OW, as performed in stapedioplastic surgery. Through this access, at the end of the surgical procedure, the final surgical steps of removal of the stapes superstructure, stapedotomy, and accommodation of the stapes prosthesis are performed.

The retroauricular access through the soft tissue is similar to that commonly used in the surgery for MEI and DACI. The retroauricular access through the bone starts with a partial mastoidectomy, limited to the superior cell groups. The tegmen, anterior epitympanum, the anterior-superior wall of the external ear canal (EAC), the malleus head, and the body of the incus are identified. The wall of the EAC is thinned, the incus is removed after being disjointed from the stapes, and the bone above the second portion of the channel of the facial nerve is drilled until the OW can be identified (superior-anterior tympanotomy).

The fixation system is inserted and secured to the mastoid with bone screws. The artificial incus is positioned above the OW through the superior-anterior tympanotomy. The ball joint can be adjusted so that the correct position for the artificial incus can be achieved.

The artificial incus has no contact with the malleus, with the edges of the tympanotomy, or with the posterior wall of the tympanic membrane. Its final position is similar to that of the natural incus (Fig. 1).We used the stapes head to find the correct position for the artificial incus. The artificial incus was placed in the same position as the natural incus (Fig. 2A).

The drilling for the receiver coil, implant electronics, and the connecting cable is similar to that used in cochlear implant surgery. The removal of the stapes superstructure, the stapedotomy and the insertion of the stapes prosthesis are performed via the ear canal at the end of intervention (Fig. 2B). Table 1 presents the main steps of the Haeusler and Lenarz procedure and our procedure, whereas in Table 2 , we show the main differences between the two procedures $[8,9]$.

\section{RESULTS}

We successfully implanted the device in the five anatomical samples, following the new surgical procedure. All surgical steps were found to be fast and simple to execute. The average time for placement of the DACI in the temporal bones following this new procedure, including the dissection of the soft tissues and the suture, was 1 hour and 52 minutes (range, 1 hour and 34 minutes to 2 hour and 22 minutes). Large differences were not 
Table 1. Description of the main steps of the Haeusler and Lenarz procedure and our procedure

\begin{tabular}{|c|c|}
\hline Haeusler and Lenarz procedure & Our procedure \\
\hline Retroauricolar skin incision & Retroauricolar skin incision \\
\hline \multirow[t]{2}{*}{ Mastoidectomy } & Little mastoidectomy in epitimpanic area \\
\hline & Transcanal access to visualize the stapes \\
\hline Enlarge posterior tympanotomy & Small anterior-superior tympanotomy \\
\hline \multicolumn{2}{|l|}{$\begin{array}{l}\text { If the stapes is not well visualized, drill a part of the posterior canal wall } \\
\text { or transcanal access }\end{array}$} \\
\hline \multicolumn{2}{|l|}{ Remove the crura of the stapes and generate a hole in the footplate } \\
\hline & Remove the incus \\
\hline Prepare the bone bed and place the fixation system & Prepare the bone bed and place the fixation system \\
\hline Prepare the bone bed and place the processor & Prepare the bone bed and place the processor \\
\hline $\begin{array}{l}\text { Place the device in the mastoid with the artificial incus through the } \\
\text { posterior tympanotomy, orthogonal at the incus }\end{array}$ & $\begin{array}{l}\text { Place the device in the mastoid with the artificial incus through the anterior- } \\
\text { superior tympanotomy, in place of the incus }\end{array}$ \\
\hline Place the stapes piston (through the posterior tympanotomy) & $\begin{array}{l}\text { Remove the crura, make the hole in the footplate and place the stapes } \\
\text { piston (through the canal) }\end{array}$ \\
\hline
\end{tabular}

The main difference between the two procedures are shown in bold.

Table 2. Main differences between the two procedures

\begin{tabular}{lll}
\hline Main differences & \multicolumn{1}{c}{ Haeusler and Lenarz procedure } & Our procedure \\
\hline Time spent for surgery & Approximately $4 \mathrm{hr}$ & Approximately 2 hr \\
Risk of facial and corda tympani damage & Low (risk of a large posterior tympanotomy) & Very low (posterior tympanotomy is not performed) \\
Risk of cochlear damage & High (the vestibule stays open for approximately & Very low (the vestibule stays open for a few \\
& 30 min to $1 \mathrm{hr}$, the hole in the footplate is made & seconds or minutes, the hole in the footplate is \\
before placing the device) & made during a normal stapedioplasty)
\end{tabular}

observed among the five temporal bones. In the 3 large mastoids, our procedure was simpler than that in the 2 small temporal bones. The learning curve was crucial, as in fact the last two surgical procedures were the shortest.

\section{DISCUSSION}

The DACI system is innovative as it transmits the vibrations directly to the labyrinth through a stapes prosthesis, completely bypassing the ossicular chain. The DACI system was designed to be used in patients with severe-to-profound mixed hearing loss. For this indication, the DACI system is significantly applicable, as it constitutes the only alternative to the cochlear implant, and is much less invasive [8-10,13]. The clinical trials conducted with the DACI system to date appear to demonstrate that the risk to the cochlea is comparable to that of a stapedioplasty, while suggesting it may be necessary to investigate the procedure in a larger number of patients, with longer term follow-up, to fully assess the effects of vibration of the prosthesis in the labyrinth [8-10]. Given the relatively recent implementation, DACI placement surgery is still evolving, even with regard to some relevant aspects of the surgical technique. The surgical technique practiced by Lenarz et al. [9] differs from that originally indicated by Hausler et al. [8] in terms of the use of a single stapes prostheses, a wider mastoidectomy, and accessing the OW/middle ear through a combined transmastoid and transcanal approach in cases where the anatomy of the mastoid does not allow a good view of the OW through the posterior-inferior tympanotomy alone. These changes have simplified the technique used and reported by Hausler et al. [8] somewhat, and have also enhanced its safety. Nevertheless, the adaptations described by Lenarz et al. [9] have highlighted the difficulties and the long time required for this surgical intervention.

The modifications to the surgical technique proposed in this paper are designed to contribute further to the simplification of the surgical technique and to the reduction of clinical risk and surgical time by decreasing the risk of facial damage, both in normal anatomy and in patients with facial nerve or inner ear anomalies. The main aspects of the changes are the combined transmastoid and transcanal access to the OW, the partial mastoidectomy, anterior-superior tympanotomy, the removal of the incus, and the stapedotomy with a single stapes prosthesis anchored on the artificial incus. The combined access to the OW allows performance of the stapedotomy through the ear canal, with the best view of the OW area. Bone drilling is limited to a portion of the posterior-superior tympanic frame as in classical stapedial plastic surgery. This, due to its small size, does not involve reconstruction with cartilage, as in operations with combined access performed by Lenarz et al. [9]. Mastoid drilling is limited to the superior cell groups and to the anterior epitympanum. In our experiment on the temporal bone, the removal of 
the incus and the anterior-superior tympanotomy allowed easy access to the OW for the introduction of the actuator, under good control for the positioning of the artificial incus. This transmastoid access, which ends with a anterior-superior tympanotomy, differs from those practiced by Hausler et al. [8] and by Lenarz et al. [9,10], which require a rather large posterior-inferior tympanotomy. The type of access proposed in this paper, however, involves the removal of the incus.

Hausler et al. [8] used the incus for the placement of a second stapes prosthesis in the OW, in addition to the stapes prosthesis anchored on the artificial incus. The functional results show that the contribution of the second stapes prosthesis is insignificant compared to that of the stapes prosthesis placed on the artificial incus. None of the later surgeries practiced by Lenarz et al. $[9,10]$ used the second stapes prosthesis in the OW, thereby reducing the cochlear risk as a consequence of the stapedectomy. On the other hand, the presence of both the natural incus and artificial incus occupies space near the OW, complicating the positioning and the stapedotomy. The presence of the natural incus, therefore, does not seem to play a favorable role in DACI placement and/or performance outcomes. Even in the event of a DACI failure, and a decision to opt for a CI surgery, it would be hard to conceive of a possible role for the natural incus. As stated in the instructions for use, the partial removal of the incus may be required as part of the surgical technique for implanting the DACI.

In conclusion, the purpose of our study was to examine the feasibility of an amended DACI placement procedure to both facilitate and expedite the standard procedure currently used. We tested this procedure on a small number of samples (five temporal bones), similar to the number of samples originally reported by Hausler et al. [8] for the minimally invasive cochlear implant technique. Our proposal for an amended DACI surgical procedure takes into consideration the combined access to the OW, transcanal access for the stapedotomy, and transmastoid access with the removal of the incus, and an anterior-superior tympanotomy for accommodation of the actuator and the artificial incus. This simplified the surgical procedure and consequently reduced the time required for DACI placement, and it reduced the risk of facial and corda tympani damage; however, the actual effects should be verified in patients.

\section{CONFLICT OF INTEREST}

No potential conflict of interest relevant to this article was reported.

\section{REFERENCES}

1. Baumgartner WD, Boheim K, Hagen R, Muller J, Lenarz T, Reiss S, et al. The vibrant soundbridge for conductive and mixed hearing losses: European multicenter study results. Adv Otorhinolaryngol. 2010;69:38-50.

2. Beltrame AM, Martini A, Prosser S, Giarbini N, Streitberger C. Coupling the Vibrant Soundbridge to cochlea round window: auditory results in patients with mixed hearing loss. Otol Neurotol. 2009 Feb;30(2):194-201.

3. Bernardeschi D, Hoffman C, Benchaa T, Labassi S, Beliaeff M, Sterkers $\mathrm{O}$, et al. Functional results of Vibrant Soundbridge middle ear implants in conductive and mixed hearing losses. Audiol Neurootol. 2011 Sep;16(6):381-7.

4. Bruschini P, Beltrame AM, Colletti V. Le protesi impiantabili dell' orecchio medio. In: Proceedings of Aggiornamenti Monografici $31^{\circ}$ Convegno Nazionale di Aggiornamento; 2007 Oct; Cernobbio, Italy.

5. CollettiV, Soli SD, Carner M, Colletti L. Treatment of mixed hearing losses via implantation of a vibratory transducer on the round window. Int J Audiol. 2006 Oct;45(10):600-8.

6. Dumon T, Gratacap B, Firmin F, Vincent R, Pialoux R, Casse B, et al. Vibrant Soundbridge middle ear implant in mixed hearing loss. Indications, techniques, results. Rev Laryngol Otol Rhinol (Bord). 2009;130(2):75-81.

7. Martin C, Deveze A, Richard C, Lefebvre PP, Decat M, Ibanez LG, et al. European results with totally implantable carina placed on the round window: 2-year follow-up. Otol Neurotol. 2009 Dec;30(8): 1196-203

8. Hausler R, Stieger C, Bernhard H, Kompis M. A novel implantable hearing system with direct acoustic cochlear stimulation. Audiol Neurootol. 2008 Jun;13(4):247-56.

9. Lenarz T, Zwartenkot JW, Stieger C, Schwab B, Mylanus EA, Caversaccio M, et al. Multicenter study with a direct acoustic cochlear implant. Otol Neurotol. 2013 Sep;34(7):1215-25.

10. Lenarz T, Verhaert N, Desloovere C, Desmet J, D'hondt C, Gonzalez $\mathrm{JC}$, et al. A comparative study on speech in noise understanding with a direct acoustic cochlear implant in subjects with severe to profound mixed hearing loss. Audiol Neurootol. 2014 Jul;19(3):16474.

11. Bernhard H, Stieger C, Perriard Y. Design of a semi-implantable hearing device for direct acoustic cochlear stimulation. IEEE Trans Biomed Eng. 2011 Feb;58(2):420-8.

12. Hausler R. Cochlear implantation without mastoidectomy: the pericanal electrode insertion technique. Acta Otolaryngol. 2002 Oct; 122(7):715-9.

13. Zwartenkot JW, Snik AF, Kompis M, Stieger C. Gain and maximum output of implantable hearing devices in patients with moderate to severe sensorineural hearing loss. J Hear Sci. 2012;2(2):35-40. 